\title{
Anti-P110 Autoantibodies Identify a Subtype of "Seronegative" Myasthenia Gravis with Prominent Oculobulbar Involvement
}

\author{
Flavia Scuderi, Mariapaola Marino, Lucrezia Colonna, Francesca Mannella, \\ Amelia Evoli, Carlo Provenzano, and Emanuela Bartoccioni \\ Institutes of General Pathology (FS, MM, LC, FM, CP, EB) and Neurology (AE), Catholic University, Rome, Italy
}

\begin{abstract}
SUMMARY: Myasthenia gravis (MG) is an autoimmune disease characterized by muscle weakness and pathogenetic autoantibodies directed against the nicotinic acetylcholine receptor (seropositive myasthenia gravis; SPMG). Nearly $15 \%$ to $20 \%$ of MG patients do not have these antibodies (seronegative myasthenia gravis; SNMG), but several evidence indicate that these patients have circulating pathogenic autoantibodies directed against other muscle antigens. Using the TE671 rhabdomyosarcoma cell line as an antigen source, we analyzed sera from 63 SNMG and 26 SPMG patients and 26 healthy blood donors by FACS analysis. We found that 40 of 63 SNMG patients and only 1 of 26 SPMG patients had IgG binding to the TE671 cell line. None of the sera bound to the unrelated MRC5 cell line. To identify the antigen, we analyzed sera immunoreactivity in more detail by immunoprecipitation of biotinylated membrane proteins from TE671 cells. When the immunoprecipitated proteins were separated by SDS-PAGE electrophoresis and then transferred to nitrocellulose membranes, we found that SNMG IgG identify a band corresponding to a protein with a molecular weight of $110 \mathrm{kDa}(\mathrm{P} 110)$, which is not recognized by seropositive MG sera. This anti-P110 immunoreactivity is significantly associated with a distinct clinical picture characterized by a prominent involvement of ocular and bulbar muscles, with frequent respiratory problems $(p<0.005)$, and is recognized by a specific antimuscle specific kinase (MUSK) antiserum. In a recent article, the presence of anti-MuSK antibodies was described in SNMG. Our results confirm the presence of these antibodies in SNMG and suggest that anti-P110/MuSK autoantibodies identify a subtype of SNMG in which the different pathogenesis induces the distinct clinical picture. (Lab Invest 2002, 82:1139-1146).
\end{abstract}

$M$ yasthenia gravis (MG) is a neuromuscular transmission disease arising from antiacetylcholine receptor (AChR) autoantibodies. These antibodies can be detected in the patients' sera with a radioimmunoassay, using human antigen labeled with ${ }^{125} \mathrm{I} \alpha$-bungarotoxin (seropositive myasthenia gravis; SPMG) (Bartoccioni et al, 1980; Lindstrom, 1977). Anti-AChR antibody is not detected with this test in $15 \%$ to $20 \%$ of patients with generalized MG (seronegative myasthenia gravis; SNMG) (Evoli et al, 1989; Mossman et al, 1986). These SNMG patients frequently complain of severe prominent ocular and bulbar weakness, which shows an unsatisfactory response to oral anticholinesterases, although improving with plasmapheresis and immunosuppressive therapy (oculobulbar SNMG) (Evoli et al, 1996). It has been shown that plasma or purified immunoglobulins from SNMG patients can transfer the neuromuscular transmission defect in mice (Burges et al, 1994; Mossman et al, 1986; Provenzano et al, 1988). Blaes and coworkers (2000) have shown that IgG from some

DOI: 10.1097/01.LAB.0000028144.48023.9B

Received February 13, 2002.

Financial support was from Catholic University grants to $C P$ and $E B$.

Address reprint requests to: Dr. Emanuela Bartoccioni, Institute of General Pathology, Catholic University, Largo Francesco Vito 1, 00168 Rome, Italy.E-mail:ebartoc@rm.unicatt.it
SNMG patients bind to a surface antigen, distinct from the $\mathrm{ACh} \bar{R}$, on the TE671 rhabdomyosarcoma cell line. These observations, along with the clinical characteristics, reinforce the hypothesis that SNMG is an antibody-mediated autoimmune disease, which should be distinguished from SPMG.

In the present article, we used a cytofluorometric assay similar to that of Blaes et al (2000) to study a large MG population, and we correlated the laboratory findings with clinical data: we found a high frequency of positive results in SNMG, whereas TE671-specific immunoreactivity was absent in most SPMG patients. Moreover, both the greatest values of specific immunoreactivity and the highest positivity rate were found in those patients with prominent oculobulbar involvement. The antibody titer also showed a positive correlation with disease severity, with modifications that paralleled the clinical course along with the immunosuppressive or plasmapheresis treatment.

To look for a candidate antigen, we used patients' sera to immunoprecipitate putative target antigens from surface-biotinylated TE671 cell lysates. We found a highly significant association between the presence of autoantibodies directed against a membrane protein with a molecular weight of about 110 kDa (P110) and oculobulbar SNMG. It has recently been reported that antibodies directed against the muscle-specific receptor tyrosine kinase (MuSK) had been detected in the sera of SNMG patients (Hoch et 
al, 2001): we found that P110 (as precipitated by patients' sera) and the MuSK band (as precipitated by a goat antiserum) have the same apparent electrophoretic mobility; moreover, the $\mathrm{P} 110$ protein is recognized by a specific anti-MuSK antiserum. Our findings first confirm the presence of anti-MuSK antibodies in SNMG and, when examined along with the peculiar clinical characteristics, further support the identification of oculobulbar SNMG as a defined disease entity in which the different autoantibodies determine this distinct clinical picture.

\section{Results}

We tested sera from 26 SPMG patients, 63 SNMG patients, 26 healthy blood donors (HBD), and 19 subjects with other neuromuscular diseases (OND) for IgG binding to TE671 by fluorescence-activated cell sorted (FACS) analysis. HBD sera showed a very low reactivity, close to blank value (Fig. 1a). Positivity cut-off (2.10 arbitrary units [AU]) was determined as the mean +2 SD of HBD AU (mean 0.92; SD 0.59). When patients' sera were tested, only 1 of 26 SPMG patients had a positive result, whereas 40 (63.5\%) of 63 SNMG patients had positive results (Fig. 2). To verify how specific this immunoreactivity was, we repeated the assay on the MRC5 fibroblastoid cell line: 10 of 10 SNMG, 2 of 2 SPMG, and 5 of 5 HBD sera did not show any reactivity with this cell line (Fig. 1b). Among OND patients, 9 of 11 polymyositis and 5 of 8 mitochondrial myopathies showed positive results on TE671 by FACS analysis. Thus, although this test is not specific when applied to other nonmyasthenic neuromuscular diseases, in MG it shows a definite association with the seronegative form of this disease.
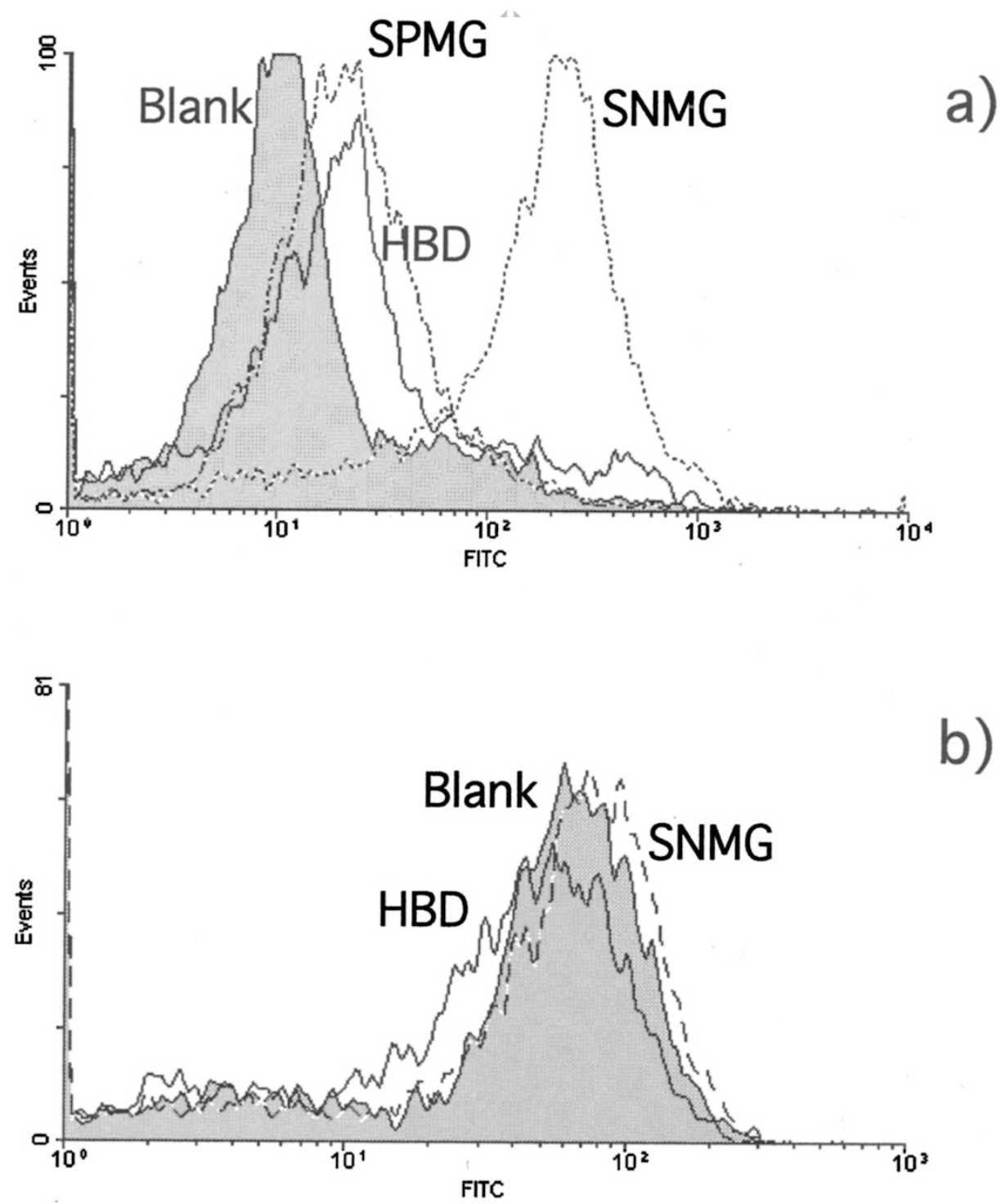

Figure 1.

Fluorescence-activated cell sorted (FACS) analysis of IgG binding to TE671 (a) and to MRC5 (b) cells. The characteristic patterns of a blank reading (second antibody alone), a control serum (healthy blood donor; $H B D$ ), a seropositive myasthenia gravis serum (SPMG; anti-acetylcholine receptor [AChR] antibody titer: 17.5 nM), and a seronegative myasthenia gravis serum (SNMG; class 4 according to Osserman's classification, with oculobulbar symptoms) are shown. 


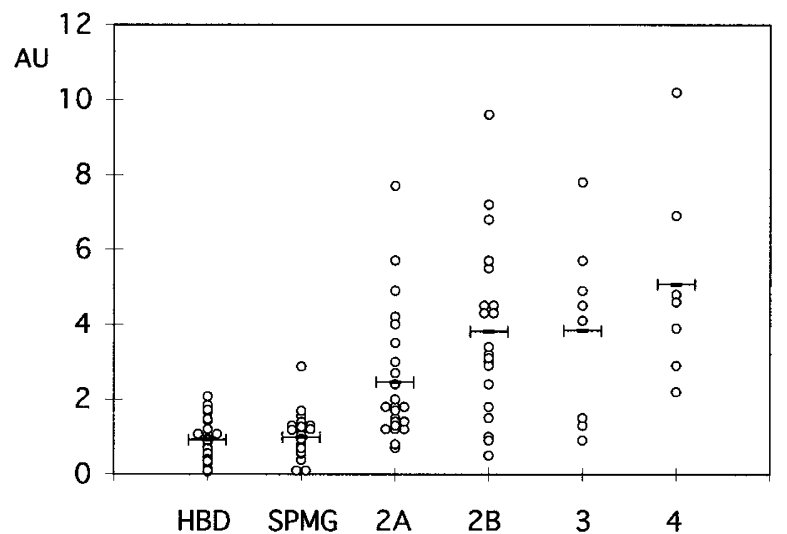

Figure 2.

Distribution of anti-TE671 reactivity (arbitrary units [AU]) in HBD, SPMG, and SNMG patients grouped according to Osserman's classification (2A, 2B, 3, and 4). Mean values for each group are indicated by a horizontal bar.

We evaluated the possible correlation of these results with SNMG severity: both the percent positivity and the intensity of immunoreactivity correlated with disease severity $(35 \%, 76 \%, 67 \%$, and $100 \%$ for Osserman's grades 2A, 2B, 3, and 4, respectively; the mean intensity is indicated in Fig. 2). Immunoreactivity was more frequent $(29 / 34$ versus $11 / 29 ; 85 \%$ versus $38 \% ; p<0.0001$; Fig. 3) and with higher mean values $(4.3 \pm 2.3$ versus $2.3 \pm 1.7 \mathrm{AU}, p<0.001)$ in SNMG with prominent oculobulbar involvement than in the other SNMG patients.

No correlation was observed when gender, age at onset, or immunosuppressive therapy at the time of serum sample collection where considered (data not shown). When we studied the correlation between individual clinical follow-up and TE671/immunoreactivity levels in seven of these patients, we found that a worsening of clinical symptoms was associated with an increase in immunoreactivity levels, whereas an amelioration corresponded to a decrease. In Figure 4 we show an example for one of these patients, where it is evident that changes in

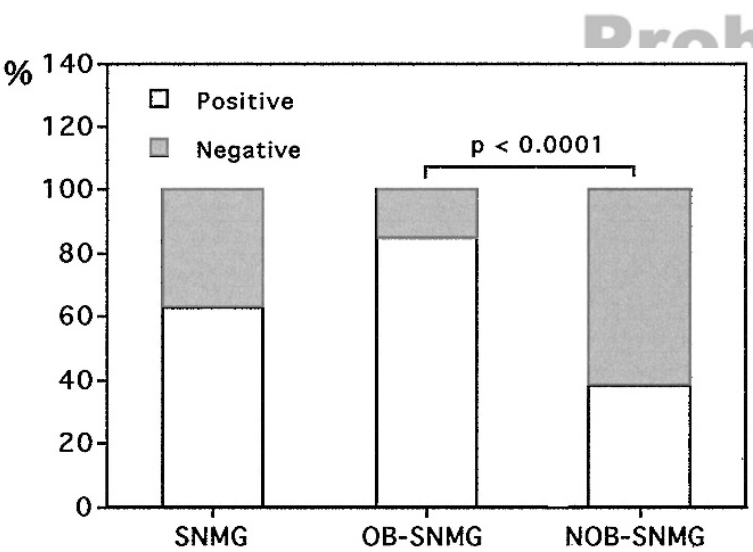

Figure 3.

Distribution of anti-TE671 reactivity in seronegative myasthenic patients: total (SNMG), with prevalent oculobulbar symptoms (OB-SNMG), and without prevalent oculobulbar symptoms (NOB-SNMG) populations are indicated. Statistical significance for OB-SNMG versus NOB-SNMG, $\chi^{2}$ test: $p<0.0001$.

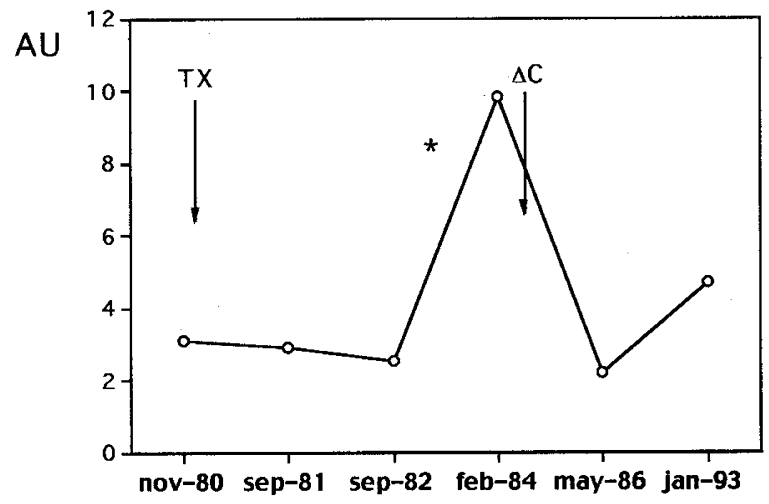

Figure 4.

Serial determinations of anti-TE671 immunoreactivity in a SNMG patient with prevalent oculobulbar symptoms. The elapse of time is not drawn to scale. TX $=$ thymectomy; ${ }^{*}=$ period when a clinical deterioration started to appear, with respiratory failure requiring mechanical ventilation and tracheostomy; $\Delta C=$ time when corticosteroid treatment was started along with a course of plasma exchanges after which the patient progressively improved.

clinical status were associated with modifications of anti-TE671 immunoreactivity.

To look for a candidate antigen, we analyzed sera immunoreactivity in more detail by immunoprecipitation of biotinylated membrane proteins from TE671 cells. We tested the sera from 21 patients with SNMG, 2 with SPMG, 2 with polymyositis, 2 with mitochondrial myopathies, and 4 HBD. When the immunoprecipitated proteins were separated by SDS-PAGE electrophoresis and then transferred to nitrocellulose membranes, we observed the patterns shown in Figure 5 ( $\mathrm{A}$ and $\mathrm{B}$ ). We found that a band corresponding to a protein of molecular weight of about $110 \mathrm{kDa}$ (P110) was present only in the immunoprecipitates from SNMG sera and particularly from those with prominent oculobulbar symptoms, while it was always absent in the immunoprecipitates from all other sera, regardless of the original disease.

\section{A \\ SP SP OB OB OB HBD}

P110

B

\section{OB NOB SP SP OB HBD}

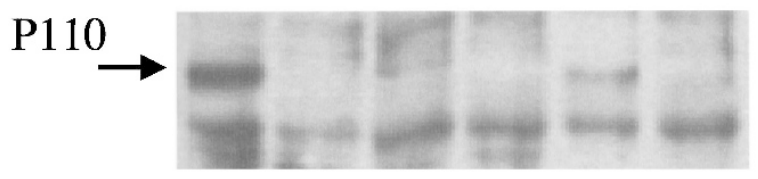

Figure 5.

Blots of biotinylated membrane proteins immunoprecipitated by patient sera. A, Comparison of two SPMG patient sera with three OB-SNMG patient and one HBD serum. B, Comparison of two OB-SNMG patient sera, with one NOBSNMG serum and two SPMG patient sera and one HBD. 
When we correlated the positivity for anti-P110 antibodies with the clinical characteristics of SNMG patients, we found a strong correlation with the presence of oculobulbar symptoms: 11 of 12 SNMG patients with these symptoms showed the P110 band at the immunoblot, while only 2 of 9 SNMG patients without these symptoms had the same result (Fisher's exact test: $p<0.005$; Fig. 6).

After searching protein databases and literature, we found that a possible candidate (a protein that must be highly represented at the neuromuscular junction and with a molecular weight around $110 \mathrm{kDa}$ ) could be the muscle-specific kinase MuSK (Hoch, 1999; Meier et al, 1997; Ruegg, 2001). We used the same biotinylation and immunoprecipitation assay to verify the presence of MuSK on TE671 cell membranes and whether the electrophoretic patterns, obtained by using patients' sera or an anti-MuSK antiserum, produced bands of identical molecular weight. In Figure 7 we show that TE671 cells express a protein that is recognized by an anti-MuSK antiserum and that both SNMG sera and anti-MuSK antiserum immunoprecipitated a protein with the same apparent molecular weight, while the HBD control serum did not show such a band. We confirmed the presence of MuSK in the P110 immunoprecipitated by SNMG sera by stripping off and reprobing blots with rabbit anti-MuSK antiserum: the results are shown in Figure 8 in which sera from patients with polymyositis, and from SNMG patients with prevalent oculobulbar symptoms, and without prevalent oculobulbar symptoms and sera from HBD have been used. The polymyositis serum recognized a protein with a similar but lower molecular weight, which is aspecifically recognized by the secondary antibody.

\section{Discussion}

In its classical form, MG is determined by antibodies aimed at the postsynaptic AChR. In a restricted group of patients, it is not possible to detect these antibodies. Experimental and clinical data have long supported the view that the anti-AChR-negative form of

\section{HBD OB MuSK}

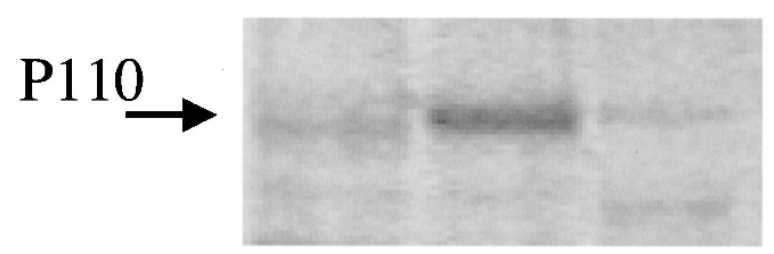

Figure 7.

Blots of biotinylated membrane proteins as immunoprecipitated by human sera from an HBD or from an OB-SNMG patient $(O B)$ or by an anti-muscle specific receptor tyrosine kinase antiserum (MUSK).

\section{PM NOB OB OB OB HBD}
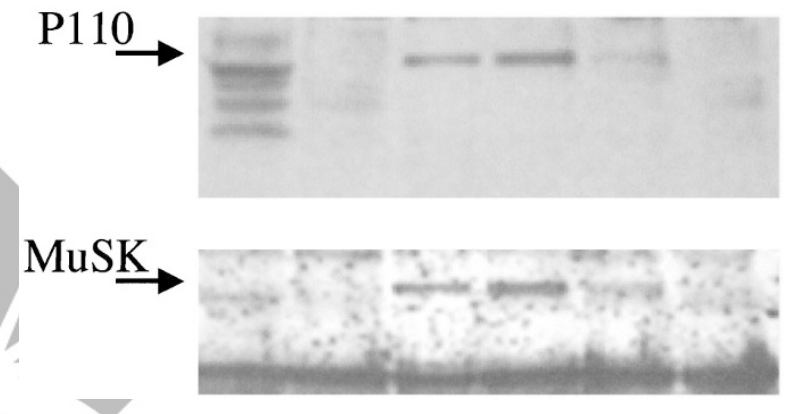

Figure 8.

Blots of biotinylated membrane proteins immunoprecipitated by patient sera. Top, Comparison of one polymyositis patient serum (PM) with one NOBSNMG (NOB), three OB-SNMG $(O B)$, and one HBD sera. Bottom, The same blot after stripping off the HRP-conjugated avidin and reprobing with an anti-MuSK rabbit antiserum followed by a goat anti-rabbit HRP-conjugated antiserum.

$M G$ is a disease entity that should be distinguished from the anti-AChR-positive one: albeit plasma or purified lgG can transfer the neuromuscular transmission defect to mice, no antibody bound to the mouse AChRs and only a limited muscle AChR loss have been observed (Burges et al, 1994; Mossman et al, 1986; Provenzano et al, 1988); moreover, patients respond well to immunosuppressive drugs and plasma exchange while getting generally limited benefit from oral anticholinesterase therapy (Evoli et al, 1989)

Despite the many efforts, the antigen(s) and patho-

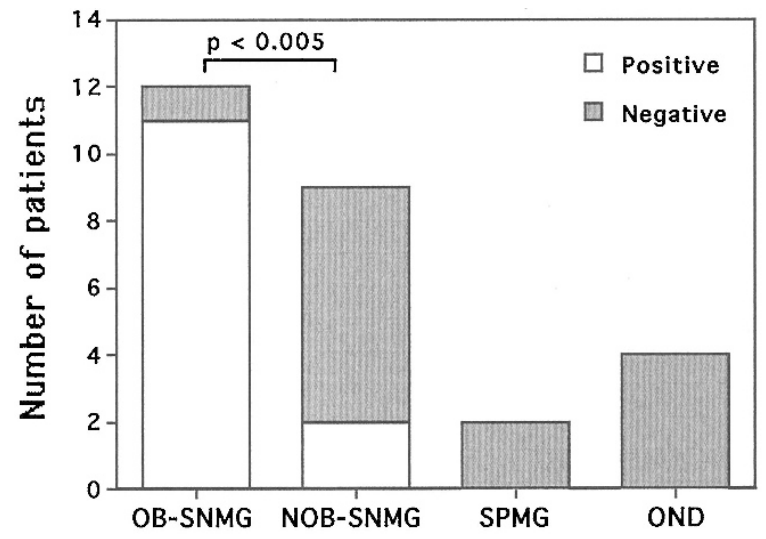

Figure 6.

Distribution of anti-P110 reactivity. Statistical significance for OB-SNMG versus NOB-SNMG, Fisher's exact test: $p<0.005$. genetic mechanism(s) involved in this form of disease have not been definitively identified. Blaes et al (2000), by means of a cytofluorometric assay, found that IgG from SNMG sera bind to a surface antigen(s) of the TE671 cell line but not to fibroblastoid cells transfected with human AChR. This test gave the same results even when the TE671 cells, which express the fetal AChR (the one containing the $\gamma$ subunit), were transfected with the adult $(\epsilon)$ subunit of $A C h R$, further proving that SNMG antibodies bind to a muscle surface antigen distinct from the AChR. SPMG sera were always negative, as in our experiments, probably because TE671 cells express very little surface AChRs, because they are mostly retained intracellularly (Conroy et al, 1990).

In the present study, we confirm the positive results of the FACS assay in SNMG patients, and we start to 
characterize the antigen recognized by patients' immunoglobulins. With this assay we have been able to detect a definite immunoreactivity against TE671 cells. The surface antigen(s) is not bound by HBD sera, while it is recognized by $63.5 \%$ of SNMG sera, by only 1 of 26 SPMG sera, and by 14 of 19 patients with OND. When the MRC5 fibroblastoid cell line was used, no immunoreactivity was present in any serum we tested; moreover, when we used primary human myoblasts as the antigen source, we had results that can be superimposed to the ones obtained with TE671 cells (data not shown), further proving that we are detecting an IgG population against a muscle-specific antigen(s). The large variety of muscle antigens, and of antimuscle antibodies most likely present in the OND group, can be the basis of the positive results observed in these patients. Despite this, when only the MG population is considered, the immunoreactivity against TE671 cells seems to be specific for SNMG. Previous observation had pointed out that SNMG shows clinical heterogeneity and that a subgroup of these patients is characterized by prevalent oculobulbar involvement and by a more stable natural course (Evoli et al, 1996). When we compared the results of our cytofluorometric test in SNMG patients with their clinical status, we found a positive correlation of both the percent positivity and the intensity of immunoreactivity with disease severity (Fig. 2). In each group, the most severely affected patients are those with oculobulbar symptoms: these patients have the strongest association with immunoreactivity against TE671 cells $(p<0.0001$; Fig. 3). Our finding that $63.5 \%$ of SNMG are positive with TE671 cells assay agrees with the laboratory observations of Blaes et al (2000). The highly statistically significant correlation between our FACS analysis data and the clinical picture, unavailable in previous articles, further supports the heterogeneity in the pathogenesis of SNMG, which is then reflected in the different clinical picture.

The SNMG specificity of the immunoreactivity we are detecting with this FACS assay is further proved by the analysis of serial samples from single patients. By this analysis, we found that disease severity and antibody titer had a parallel course: a worsening of clinical symptoms was associated with an increase in antibody levels, and an amelioration of symptoms (due to either plasma exchange or immunosuppressive therapy) was accompanied by a decrease in the antibody titer (Fig. 4). Although the latter can be ascribed to a generalized immunosuppression, which lowers any antibody, the former is a clear index of the SNMG specificity of the immunoreactivity we are detecting: there is no reason for an irrelevant antibody to increase along with a worsening of symptoms.

The second step in our work was to try to identify the fine specificity of these serum antibodies. To reach this goal, we used immunoprecipitation of biotinylated membrane proteins as the preferential approach. This method has the advantage of a great sensitivity coupled with selectivity for membrane proteins (Schuberth et al, 1996), which are essential requirements. As a matter of fact, previous works could not demon- strate any difference between SNMG patient and healthy control sera using a crude normal human muscle extract as antigen source by means of a quantitative immunoblotting technique (Sharshar et al, 1998). The method we used is more selective because the antigens we analyzed are only the membrane ones and because it adds an amplification step with the avidin-conjugated horseradish peroxidase (HRP) plus the enhanced chemiluminescence (ECL) detection. With this approach, we identified a $110-\mathrm{kDa}$ membrane protein $(\mathrm{P} 110)$ as the putative antigen, because it was precipitated only by SNMG immunoglobulins (Fig. 7). It is impressive that 11 of 12 SNMG patients with oculobulbar involvement, and only 2 of 9 without oculobulbar involvement, showed this antibody specificity (Fig. 6), which suggests that the antigen we are detecting with the FACS assay and the one with the immunoblot are likely to be the same.

Recently, Hoch and coworkers have shown that anti-MuSK antibodies are present in anti-AChR negative myasthenia (Hoch et al, 2001) and that these antibodies inhibit the agrin-induced AChR clustering in vitro. MuSK is a plasmamembrane tyrosinekinase receptor, with a molecular weight of $110 \mathrm{kDa}$ (Hoch, 1999). Our data show that TE671 cells, indeed, express a membrane protein of the correct molecular weight, which is immunoprecipitated by an anti-MuSK antiserum. Moreover, the P110 band precipitated by patients' sera is recognized by an anti-MuSK antiserum, which further supports the hypothesis that the protein we are detecting is MuSK. Kinase function of MuSK is activated upon binding with nerve-released agrin and mediates AChR clustering and neuromuscular junction formation (Glass et al, 1996; Herbst and Burden, 2000). Plastic modifications of synaptic organization is an active process requiring a continuous cross-talk between the presynaptic nerve ending and the postsynaptic muscular apparatus (Dai and Peng, 1998): anything interfering with MuSK activity or postsynaptic membrane integrity, such as patients' antibodies or complement-mediated lysis, can modify synapse structure and/or function and therefore impair neuromuscular transmission.

It has been suggested that most anti-AchR-negative MG patients seem to have distinct clinical characteristics in that weakness can be prevalent or even limited to ocular, facial, and pharyngolaryngeal muscles, and the response to oral anticholinesterases can span from scarce benefit to overt intolerance (Evoli et al, 1989). Our findings, although not formally proving that anti-MuSK antibodies induce this form of MG, can constitute an experimental support to the definition of the pathogenetic mechanisms involved in this disease and to the elucidation of the processes involved in synapse formation and maintenance. This will represent a substantial contribution to the identification and definition of SNMG as a new disease entity. Further in vitro and in vivo studies are underway to elucidate the exact role of these autoantibodies in the pathogenesis of SNMG. 


\section{Materials and Methods}

\section{Patients and Controls}

We studied 63 patients with generalized MG, who had repeatedly shown negative results with the conventional radioimmunoassay (SNMG). The diagnosis of MG was based on the association of typical history and signs of fluctuating muscle weakness together with electrophysiologic findings of neuromuscular transmission defect, ie, more than $11 \%$ decrement in compound muscle action potential on low-rate repetitive nerve stimulations and/or increased jitter on single fiber electromyography. Electromyography findings of neurogenic and/or myopathic disorders, compound muscle action potential increment on high-rate repetitive nerve stimulations (maximal voluntary contraction), and repetitive response to a single nerve stimulus were absent in every patient. A positive response to neostigmine im/edrophonium iv was present in all patients but five, even if the subsequent response to oral anticholinesterases was generally less satisfactory than in SPMG patients. MG severity was graded according to the method of Osserman (Osserman and Genkins, 1971). The 63 SNMG patients (40 women and 23 men), had age at onset ranging from 6 to 83 years. Clinical data are shown in Table 1. Among these SNMG patients, 34 showed prevalent oculobulbar symptoms (ophthalmoparesis, facial weakness, nasal speech, dysphagia, frequent respiratory crises), with a severe disease (grade 2B-4) in 30 of them. Twenty-two of the remaining 29 patients had a mild generalized form of disease (grade $2 \mathrm{~A}$ ). Forty of the 63 SNMG were not receiving immunosuppressive therapy at the time of serum sample collection. Control sera were from 26 SPMG patients, 19 subjects with other neuromuscular diseases (11 polymyositis and 8 mitochondrial myopathies), and 26 HBD from the local blood bank.

\section{Anti-AChR Antibody Testing}

The anti-AChR assay was performed according to Lindstrom's method, with minor modifications (Bartoccioni et al, 1980), using human muscle leg extract as antigen. All sera with a negative $(<0.5 \mathrm{~nm})$ value were repeatedly assayed at various dilutions and with different antigen extracts to confirm the absence of anti-AChR antibodies.

\section{Cell Lines}

We used the rhabdomyosarcoma TE671 and the fetal fibroblastoid MRC5 human cell lines. Both cell lines were grown in DMEM plus 10\% FCS, $1 \%$ antibiotics, and $10 \mathrm{~mm} \mathrm{L-glutamine}$ and maintained in a humidified incubator at $37^{\circ} \mathrm{C}$ with $5 \% \mathrm{CO}_{2}$.

\section{Flow Cytometry}

FACS analysis was used to detect binding of patients' IgG to the surface of TE671 and MRC5 cells according to the method of Marino et al (2001), with minor modifications: cells $\left(5 \times 10^{5}\right.$ per test) were incubated for 30 minutes in $50 \mu \mathrm{l}$ of $1: 10$ serum dilution in PBS/BSA, followed by FITC-conjugated goat antihuman IgG (Kallestad) antiserum. Cells were then analyzed on a FACScan (Profile XL; Coulter, Fullerton, California). The gate was set on total cell population and the mean fluorescence intensity was reported. Aspecific binding was detected by adding to each experiment a sample of cells incubated with FITCconjugated goat anti-human IgG second antibody/ antiserum alone (blank reading); this value was subtracted from sample mean fluorescence intensity, obtaining the binding $\mathrm{AU}$; at least one HBD and one SNMG-positive serum were assayed in each test session as quality controls.

Table 1. Clinical Characteristics of Patients with Seronegative Myasthenia Gravis

\begin{tabular}{lrrr}
\hline & Women & Men & Total \\
\hline & $40(63.5 \%)$ & $23(36.5 \%)$ & 63 \\
Age at onset & & & \\
$\leq 20$ & $9(22.5 \%)$ & $5(21.7 \%)$ & $14(22.2 \%)$ \\
$>20 \leq 40$ & $13(32.5 \%)$ & $11(47.8 \%)$ & $24(38.1 \%)$ \\
$>40$ & $18(45.0 \%)$ & $7(30.4 \%)$ & $25(39.7 \%)$ \\
Osserman classification & & & $26(41.3 \%)$ \\
$2 A$ & $13(32.5 \%)$ & $13(56.5 \%)$ & $21(33.3 \%)$ \\
$2 B$ & $16(40.0 \%)$ & $5(21.7 \%)$ & $9(14.3 \%)$ \\
3 & $6(15.0 \%)$ & $3(13.0 \%)$ & $7(11.1 \%)$ \\
4 & $5(12.5 \%)$ & $2(8.7 \%)$ & $24(38.1 \%)$ \\
Thymectomy & $12(30.0 \%)$ & $12(52.2 \%)$ & $8(12.7 \%)$ \\
Hyperplasia & 6 & 2 & $2(3.2 \%)$ \\
Thymoma & 1 & 1 & $14(22.2 \%)$ \\
Involution & 5 & 9 & $23(36.5 \%)$ \\
Corticosteroid therapy & $11(27.5 \%)$ & $9(39.1 \%)$ & $34(54.0 \%)$ \\
Oculobulbar involvement & $25(62.5 \%)$ & & \\
\hline
\end{tabular}




\section{Biotinylation, Immunoprecipitation, and Blotting}

Plasma membrane proteins were biotinylated to be easily distinguished after immunoprecipitation. Amino terminal residues of membrane proteins from TE671 cells were linked to NHS-LC-biotin (Pierce, Rockford, Illinois) (dissolved in PBS at $10 \mathrm{mg} / \mathrm{ml}$ ) according to the method of Schuberth et al (1996). Biotin was added to $8 \times 10^{6}$ cells in $1 \mathrm{ml}$ of PBS to achieve the final concentration of $100 \mu \mathrm{g} / \mathrm{ml}$. After 15 minutes at room temperature on a rotating shaker, the biotinylation was blocked with lysine (final concentration 500 $\mu \mathrm{g} / \mathrm{ml}$ ) for 5 minutes followed by three washes in PBS at $4^{\circ} \mathrm{C}$. Previous flow cytometry experiments had shown that biotin labeling did not affect the immunoreactivity of cells (our unpublished results), similarly to what was already published in a different system (Schuberth et al, 1996). The biotinylated cell pellets were lysed with $1 \mathrm{ml}$ of $1 \%$ Triton X-100 in $50 \mathrm{~mm}$ Tris- $\mathrm{HCl}, 2$ mM EDTA (pH 8), 1 mm phenylmethylsulfonyl fluoride, and $1 \mathrm{~mm}$ sodium orthovanadate (lysis buffer). After 30 minutes on ice, lysates were centrifuged at $4^{\circ} \mathrm{C}(10000 \times \mathrm{g}, 20$ minutes $)$, and the proteins from the supernatants were precleared with protein G-Sepharose (Pharmacia, Uppsala, Sweden) (60 minutes, $4^{\circ} \mathrm{C}$ ). One milligram of lysate proteins (as determined by Bio-Rad DC Protein Assay; Bio-Rad, Hemel Hempstead, United Kingdom) were precipitated with 5 $\mu \mathrm{l}$ of patients' serum or $10 \mu \mathrm{l}$ of anti-MuSK (Santa Cruz Biotechnology, Santa Cruz, California) and $10 \mu \mathrm{l}$ of protein G-Sepharose for 3 hours at $4^{\circ} \mathrm{C}$, with continuous shaking. The immunoprecipitates/pellets were washed three times with lysis buffer and then dissolved in $30 \mu \mathrm{l}$ of lysis buffer/SDS sample buffer (Tris- $\mathrm{HCl}[\mathrm{pH}$ 6.8], $10 \%$ SDS, $36 \%$ glycerine, 5\% 2-mercaptoethanol, $0.03 \%$ bromophenol-blue) (1/6 $\mathrm{v} / \mathrm{v})$. Samples were heated $\left(95^{\circ} \mathrm{C}, 5\right.$ minutes) before SDS-PAGE. Gels $(60 \times 80 \times 1 \mathrm{~mm})$ were run in a Mini Protean 3 Cell Powerpack 300 System (Bio-Rad).

After SDS-PAGE, gels were blotted with transfer buffer (30 mm Tris, $240 \mathrm{~mm}$ glycine, 20\% methanol) directly on pure nitrocellulose membrane $(0.45 \mu \mathrm{m})$ (Bio-Rad) at $330 \mathrm{~mA}$ for 1 hour in a Mini Trans-Blot Transfer Cell (Bio-Rad). Nonspecific binding sites on blots were blocked by incubation with $3 \%$ gelatin in 10 $\mathrm{mm}$ Tris $\mathrm{HCl}, 0.15 \mathrm{~m} \mathrm{NaCl}$ containing $0.05 \%$ Tween 20 (TBST) for 2 hours at room temperature on a rotating shaker. Biotinylated proteins were stained with HRPconjugated avidin (1:20,000 in TBST) and ECL detection was performed by exposition on a Kodak XAR-5 x-ray film (Rochester, New York).

\section{Stripping and Reprobing}

Removal of the HRP-conjugated avidin was achieved by incubation in stripping buffer $(62.5 \mathrm{~mm}$ Tris- $\mathrm{HCl}[\mathrm{pH}$ 6.8], 2\% SDS, and $10 \mathrm{~mm}$ 2-mercaptoethanol) for 50 minutes at $55^{\circ} \mathrm{C}$ with gentle shaking. Nonspecific binding was blocked by incubation with $5 \%$ dry milk in TBST $0.1 \% \mathrm{NaN}_{3}$ at room temperature for 2 hours with gentle shaking, and blots were reprobed with a rabbit polyclonal anti-MuSK antiserum (1:500; kindly gifted by M. A. Ruegg, Basel, Switzerland) in 3\% dry milk in TBST $0.1 \% \mathrm{NaN}_{3}$ at $4^{\circ} \mathrm{C}$ for 18 hours with gentle shaking; to detect the immunolabeled proteins, blots were incubated with goat anti-rabbit HRPconjugated antiserum (Bio-Rad) in TBST (1:10000) at room temperature for 1 hour with gentle shaking, and ECL detection was performed as described.

\section{Statistical Analysis}

For association studies, $p$ values were calculated by the Student's $t$ test, $\chi^{2}$ method, or Fisher's exact test where appropriate; $p<0.05$ was considered significant.

\section{Acknowledgements}

We gratefully acknowledge the scientific counseling of Dr. G. Pani in the biotinylation-immunoprecipitation protocol, Prof. S. Servidei and Dr. E. Ricci for the polymyositis and mitochondrial myopathy sera, Prof. G. D'Onofrio and Dr. F. Vigevani for the healthy blood donor sera, and Prof. M. A. Ruegg for anti-MuSK rabbit serum.

\section{References}

Bartoccioni E, Scuderi F, Scoppetta C, Evoli A, Tonali P, Guidi L, Bartoloni C, and Terranova T (1980). Myasthenia gravis, thymectomy and antiacetylcholine receptor antibody. J Neurol 224:9-15.

Blaes F, Beeson D, Plested P, Lang B, and Vincent A (2000). IgG from "seronegative" myasthenia gravis patients binds to a muscle cell line, TE671, but not to human acetylcholine receptor. Ann Neurol 47:504-510.

Burges J, Vincent A, Molenaar PC, Newsom-Davis J, Peers C, and Wray D (1994). Passive transfer of seronegative myasthenia gravis to mice. Muscle Nerve 17:1393-1400.

Conroy WG, Saedi MS, and Lindstrom J (1990). TE671 cells express an abundance of a partially mature acetylcholine receptor alpha subunit which has characteristics of an assembly intermediate. J Bio Chem 265:21642-21651.

Dai Z and Peng HB (1998). A role of tyrosine phosphatase in acetylcholine receptor cluster dispersal and formation. J Cell Biol 141:1613-1624.

Evoli A, Bartoccioni E, Batocchi AP, Scuderi F, and Tonali P (1989). Anti-AChR-negative myasthenia gravis: Clinical and immunological features. Clin Invest Med 12:104-109.

Evoli A, Batocchi AP, Lo Monaco M, Servidei S, Padua L, Majolini L, and Tonali P (1996). Clinical heterogeneity of seronegative myasthenia gravis. Neuromuscul Disord 6:155161.

Glass DJ, Bowen DC, Stitt TN, Radziejewski C, Bruno J, Ryan TE, Gies DR, Shah S, Mattsson K, Burden SJ, Di Stefano PS, Valenzuela DM, De Chiara TM, and Yancopoulos GD (1996). Agrin acts via a MuSK receptor complex. Cell 85:513-523.

Herbst R and Burden SJ (2000). The juxtamembrane region of MuSK has a critical role in agrin-mediated signaling. EMBO J 19:67-77.

Hoch W (1999). Formation of the neuromuscular junction: Agrin and its unusual receptors. Eur J Biochem 265:1-10. 
Hoch W, McConville J, Helms S, Newsom-Davis J, Melms A, and Vincent A (2001). Auto-antibodies to the receptor tyrosine kinase MuSK in patients with myasthenia gravis without acetylcholine receptor antibodies. Nat Med 7:365-368.

Lindstrom J (1977). An assay for antibodies to human acetylcholine receptor in patients with myasthenia gravis. Clin Immunol Immunopathol 7:36-43.

Marino M, Scuderi F, Mazzarelli P, Mannella F, Provenzano $C$, and Bartoccioni E (2001). Constitutive and cytokineinduced expression of $\mathrm{MHC}$ and intercellular adhesion molecule-1 (ICAM-1) on human myoblasts. J Neuroimmunol 116:94-101.

Meier T, Hauser MD, Chiquet M, Landmann L, Ruegg MA, and Brenner HR (1997). Neural Agrin induces ectopic postsynaptic specializations in innervated muscle fibers. J Neuroscience 17:6534-6544.

Mossman S, Vincent A, and Newsom-Davis J (1986). Myasthenia gravis without acetylcholine receptor antibody: A distinct disease entity. Lancet 18:116-119.

Provenzano C, Arancio O, Evoli A, Rocca B, Bartoccioni E, de Grandis D, and Tonali P (1988). Familial autoimmune myasthenia gravis with different pathogenetic antibodies. J Neurol Neurosurg Psychiatry 51:1228-1230.
Osserman KE and Genkins G (1971). Studies on myasthenia gravis: Review of a twenty-year experience over 1200 patients. Mt Sinai J Med 38:497-537.

Ruegg MA (2001). Molecules involved in the formation of synaptic connections in muscle and brain. Matrix Biol 20:312.

Schuberth HJ, Kroell A, and Leibold W (1996). Biotinylation of cell surface MHC molecules: A complementary tool for the study of MHC class II polymorphism in cattle. Immunol Methods 189:89-98.

Sharshar T, Lacroix-Desmazes S, Mouthon L, Kaveri S, Gajdos P, and Kazatchkine MD (1998). Selective impairment of serum antibody repertoires toward muscle and thymus antigens in patients with seronegative and seropositive myasthenia gravis. Eur J Immunol 28:2344-2354.

\section{Unauthorized Use Prohibited}

